\title{
Radiological appearances of gynaecological emergencies
}

\author{
Oran Roche • Nikita Chavan • Joseph Aquilina • \\ Andrea Rockall
}

Received: 5 October 2011 /Revised: 26 January 2012 / Accepted: 21 February 2012 /Published online: 18 April 2012

(C) European Society of Radiology 2012

\begin{abstract}
Background The role of various gynaecological imaging modalities is vital in aiding clinicians to diagnose acute gynaecological disease, and can help to direct medical and surgical treatment where appropriate. It is important to interpret the imaging findings in the context of the clinical signs and patient's pregnancy status.

Methods Ultrasound and Doppler are readily available in the emergency department, and demonstrate features of haemorrhagic follicular cysts, ovarian cyst rupture, endometriotic cysts and pyosalpinx. Adnexal torsion may also be identified using ultrasound and Doppler, although the diagnosis cannot be safely excluded based on imaging alone. Computed tomography (CT) is not routinely employed in diagnosing acute gynaecological complications. However due to similar symptoms and signs with gastrointestinal and urinary tract pathologies, it is frequently used as the initial imaging modality and recognition of features of gynaecological complications on CT is important.

Results Although MRI is not frequently used in the emergency setting, it is an important modality in characterising features that are unclear on ultrasound and CT.

Conclusion MRI is particularly helpful in identifying the site of origin of large pelvic masses, such as haemorrhagic uterine fibroid degeneration and fibroid prolapse or torsion. In this article, we review the imaging appearances of gynaecological emergencies in non-pregnant patients.
\end{abstract}

O. Roche $(\triangle) \cdot$ N. Chavan $\cdot J$. Aquilina $\cdot$ A. Rockall

St Barts \& The London NHS trust, St Bartholomew's Hospital,

West Smithfield,

London EC1A 7BE, UK

e-mail: oran.roche@bartsandthelondon.nhs.uk
Teaching points

- Ultrasonography is easily accessible and can identify lifethreatening gynaecological complications.

- Tomography scanners and computed radiography are not routinely used but are important to recognise key features.

- MRI is used for the characterisation of acute gynaecological complications.

- Recognition of the overlap in symptoms between gastrointestinal and gynaecological conditions is essential.

Keywords Gynecology · Emergencies · Ultrasonography . Doppler $\cdot$ Tomography scanners $\cdot \mathrm{X}$-Ray computed $\cdot$ Magnetic resonance imaging $\cdot$ Ovarian cysts $\cdot$ Endometriosis $\cdot$ Dermoid cyst $\cdot$ Torsion abnormality $\cdot$ Leiomyoma $\cdot$ Pelvic inflammatory disease

\section{Introduction}

Acute abdominal pain related to the gynaecological tract is a common presentation in the emergency department. One of the challenges facing clinicians is the wide range of differential diagnoses that must be considered when assessing abdominal pain. Often it can be difficult to distinguish gynaecological from gastrointestinal emergencies. In conjunction with clinical findings, various imaging modalities play an important role in diagnosing the cause of pain. In patients presenting with pain that is thought to originate in the gynaecological tract, ultrasound is usually employed as the first imaging modality as it is highly sensitive, fast and easy to access. CT is seldom used as an initial diagnostic tool in suspected gynaecological emergencies due to the risks associated with irradiating the pelvis. However, it 
may be difficult to localise the site of origin of the symptoms to the gynaecological tract due to the significant overlap in symptoms and signs with gastrointestinal pathologies, and CT may be selected as the first imaging modality. MRI is not usually used in the acute setting but may be important in characterisation of abnormalities that remain indeterminate following ultrasound or CT.

\section{Cyst Emergencies}

Functional or simple cysts

Ovarian follicles are frequently identified on ultrasound and $\mathrm{CT}$. Developing follicles are commonly seen as functioning cysts during ultrasound examination, usually grow $2 \mathrm{~mm}$ per day until ovulation and measure less than $3 \mathrm{~cm}$ in diameter. If ovulation does not occur, a follicular cyst develops and appears as an anechoic cyst with a thin wall and posterior acoustic enhancement (seen as an area of increased echogenicity posterior to the cyst). Functional or simple cysts are usually single, less than $6 \mathrm{~cm}$ in diameter and thin walled $(<3 \mathrm{~mm})$. Follicular cysts usually reabsorb within a 4- to 8 -week period [1].

The corpus luteum forms after ovulation as granulosa cells become luteinized and blood accumulates in the central cavity. Hence, corpus luteal cysts have a thicker, more echogenic wall with increased vascularity seen as peripheral blood flow on Doppler [2].

\section{Haemorrhagic ovarian cyst}

A haemorrhagic ovarian cyst is suspected if a patient presents with symptoms of acute lower abdominal pain, tenderness and in some cases ascites [2-4]. Blood tests often show normocytic anaemia with only mild elevation of inflammatory markers such as CRP and leucocytes when compared to conditions such as appendicitis. Measurement of $\beta$ hCG levels is also important in excluding ectopic pregnancy, which can have a similar presentation $[5,6]$.

A transvaginal ultrasound is often the first imaging modality in patients who are suspected of having an ovarian cyst haemorrhage. When there is an intracystic haemorrhage the characteristics of the bleed evolve with its age. In the acute stage, the haemorrhage is isoechoic in relation to the ovarian stroma and this can often be similar in appearance to an enlarged ovary. As the clot forms, with time the fibrin strands give a typical reticular fine 'lacy net' or 'spider web' pattern (Fig. 1). Sometimes a fluid debris level may also be seen and often, as the clot matures, it may attach to the wall of the cyst, giving it a thick-walled appearance $[2,5,7]$. The

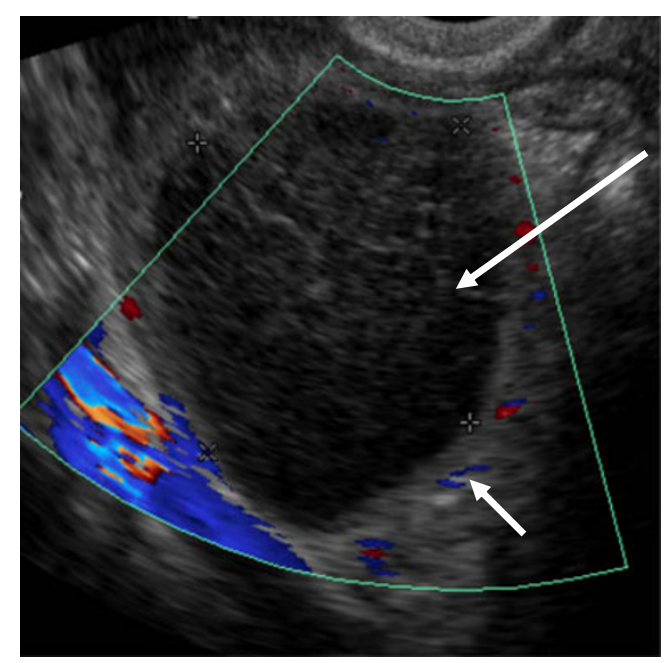

Fig. 1 Haemorrhagic cyst. Patient presented to the emergency department with acute onset of lower abdominal pain. The patient had a previous history of a right oophorectomy for an ovarian serous tumour. Transvaginal ultrasound of the left ovary demonstrates a cyst with typical lacelike reticular internal echoes (large white arrow). There is no internal blood flow but circumferential blood flow around the cyst wall is seen (small white arrow); this is a typical feature in a haemorrhagic corpus luteal cyst

use of Doppler is often used to help distinguish between malignant and benign ovarian cysts. Doppler US may demonstrate the vascular wall and the avascular internal lace-like appearances of a haemorrhagic corpus luteum cyst [8]. When performing ultrasound, it is important to exclude the presence of intraperitoneal fluid in order to exclude haemorrhagic cyst rupture. However, ultrasound has its limitations in trying to identify whether a haematoma is originating from the fallopian tube or from the ovary. In addition, the nonspecific characteristics of the presenting pain can often make CT a more attractive first investigation in the acute setting as it can exclude other intra-abdominal causes.

On CT the typical appearance of an ovarian haemorrhagic cyst is a mixed attenuation mass with a high attenuation component (45-100 HU) within the adnexa, usually with a well-defined smooth outer wall $[9,10]$. Depending on the patients' symptoms, non-contrast CT may or may not have been acquired and so the presence of high attenuation material within the cyst may be difficult to interpret, as this could represent enhancing soft tissue. However, in some cases, contrast-enhanced CT is useful in delineating the cyst wall and a delayed CT may be useful in demonstrating the pooling of contrast-enhanced blood within the pelvis in cases of cystic rupture [10].

Magnetic resonance imaging is often used when US or $\mathrm{CT}$ is unable to characterise a cystic mass and malignancy cannot be excluded. MRI shows haemorrhagic cystic lesions as bright on T1-weighted and intermediate to low on T2- 
weighted images. The age of the haemorrhage may be estimated by assessing the signal intensity of the haematoma on MRI [11-13]. For example, acute haematoma can be seen as a hypointense region in the ovary on T1-weighted images. As the haematoma becomes chronic its signal becomes bright on both T1- and T2-weighted images. Low T2 signal intensity may also be seen, particularly when haemosiderin deposition has occurred.

\section{Haemorrhagic cyst rupture}

Haemoperitoneum, a serious complication of ovarian haemorrhagic cyst rupture, may be demonstrated on both US and CT (Fig. 2a, b) and should necessitate urgent surgical intervention. In some cases, the original cyst may not be visible. On US, peritoneal or pelvic fluid is seen, with low level echoes. The cyst itself may have a crenated appearance [14]. On CT, the free peritoneal fluid has a relatively high attenuation and, on delayed CT images, there may be contrast-enhanced blood pooling in the pelvis. The limitations of CT are that it can often be difficult to identify the ovaries separately from surrounding anatomical structures. It can be challenging to distinguish whether a lesion arises from the adnexa or another structure like omentum. As ovarian haemorrhage usually affects women of reproductive age, there are also the risks associated with irradiating the pelvis [2].

On MRI, haemoperitoneum following haemorrhagic cyst rupture will be seen as variable signal intensity free peritoneal fluid with areas of both low and high signal intensity depending on the extent of blood clot formation. When clots develop, layers of low signal intensity fluid levels are often seen on T2-weighted images mixed with high signal intensity fluid. In the case of haemorrhagic ascites, the signal intensity is higher than urine on T1 and lower on T2. MRI enables the pelvic organs to be easily distinguished from each other when compared to $\mathrm{CT}$ and US. This can be advantageous when trying to assess whether the haematoma is intraovarian or extraovarian [2].

\section{Dermoid cyst rupture}

Mature cystic teratoma, also known as dermoid cyst, is the most common neoplasm of the ovary. It is derived from ectodermal differentiation of one or more germ cell layers and hence may contain hair, teeth, fat, thyroid tissue, etc. Most cystic teratomas are asymptomatic but 3\% may present as torsion and more rarely as dermoid cyst rupture. Patients present with acute pelvic pain, nausea and/or vomiting [15, $16]$.

Cystic teratomas may appear cystic with hyperechoic areas or hyperechoic densities on ultrasound with loss of through transmission [17]. CT shows a cystic mass with highly complex irregular solid components and the presence of fat within an adnexal mass is diagnostic [16].

MRI shows very high signal intensity on T1-weighted images due to the sebaceous component, which is characteristic of dermoid cyst. Fat suppression is used to differentiate fat from other haemorrhagic lesions, such as haemorrhagic cysts and endometriomas [18], which also appear hyperintense on T1-weighted images but remain high in signal intensity on fat-saturated images. If there is rupture of a cystic teratoma, there may be dispersed globules of fat within the peritoneum and signs of a chemical peritonitis with stranding in the peritoneal fat.

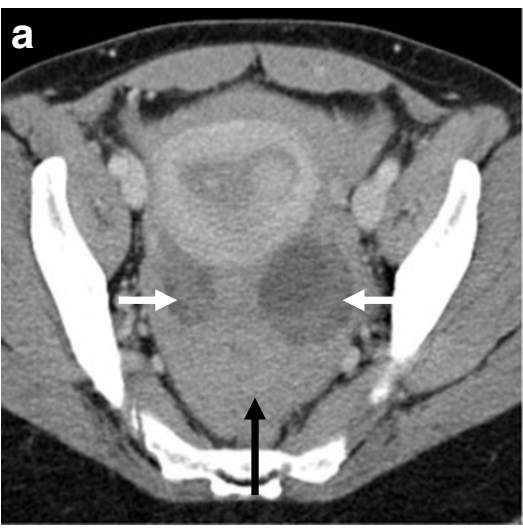

Fig. 2 Rupture of a haemorrhagic cyst. Patient presented to the accident and emergency department with a 1-day history of acute right iliac fossa pain and diarrhoea. Initially the patient was diagnosed as having an appendicitis. a CT following iv contrast administration demonstrates bilateral low-density cystic lesions (white arrows). There is extensive hyperdense free pelvic fluid representing hemorrhagic ascites (black

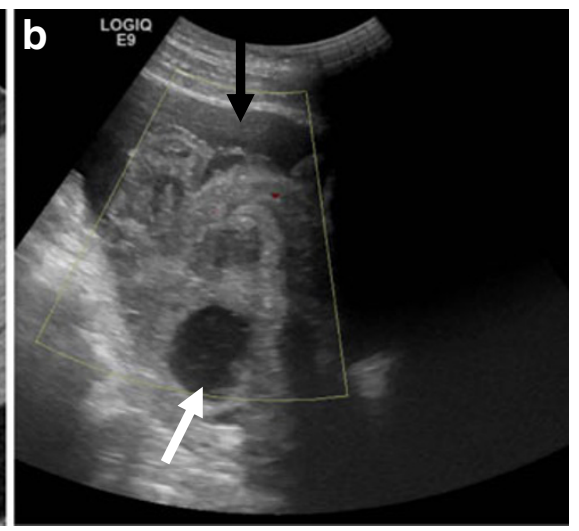

arrow). b Trans-abdominal ultrasound shows free fluid containing low level echoes in the pelvis (black arrow). There is an adnexal cyst in the pelvis representing the right haemorrhagic ovarian cyst (white arrow). The smaller right-sided cyst may be the site of rupture as the ruptured cyst may be small or difficult to visualize following rupture 

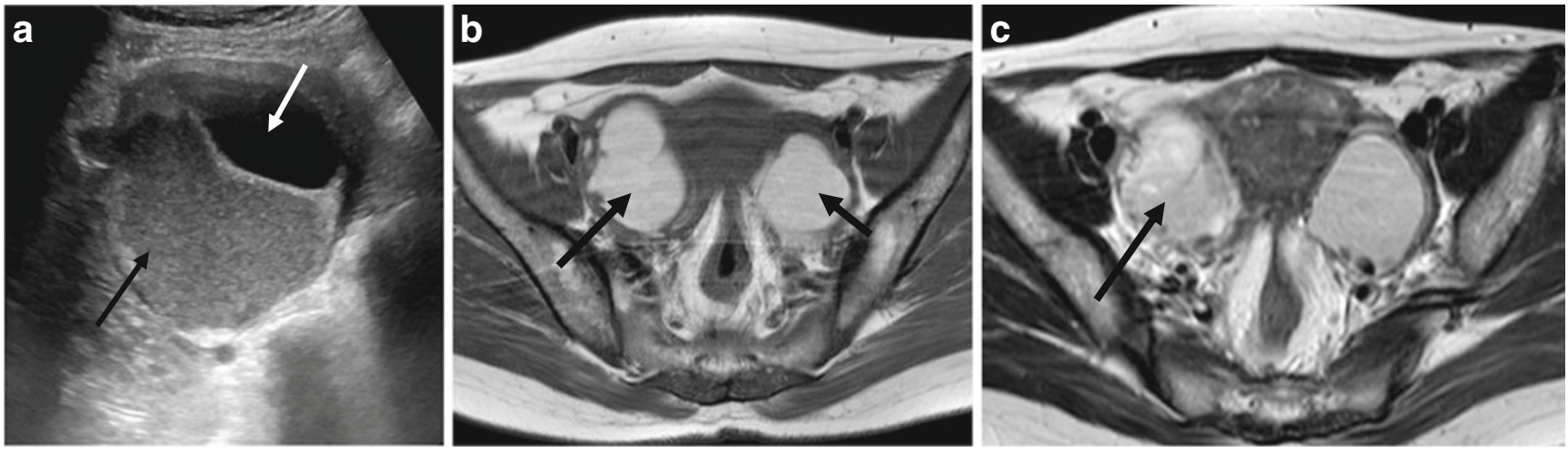

Fig. 3 Acute presentation of endometriosis. Patient presented with intermenstrual vaginal bleeding and severe lower abdominal pain. a Transvaginal ultrasound demonstrates an ovarian cyst with an area of homogeneous internal echogenicity typical of an endometriotic cyst (black arrow). There is a focal area of clot retraction along the endometriotic cyst

\section{Endometriotic cysts}

Endometriosis is caused by cyclical bleeding of hormonally responsive endometrial cells present outside the uterine lining. This can lead to complications of blood-filled cysts within the ovaries (also known as endometriomas or 'chocolate' cysts) wall (white arrow). b Axial T1 image demonstrates bilateral complex adnexal cystic masses which contain high T1 material. c Axial T2 demonstrates intermediate signal intensity with 'shading' (black arrow), typical of endometriotic blood. The appearances are in keeping with bilateral haematosalpinges in a patient with endometriosis

and haemorrhagic ascites leading to fibrosis and adhesions within the pelvis. Common presenting symptoms of this condition include chronic pelvic pain, dyspareunia, dysmenorrhoea and infertility [7, 13, 19]. Endometriosis involves the ovary in more than half the cases (up to $80 \%$ ). On ultrasound, endometriomas have homogeneous low level echogenicity
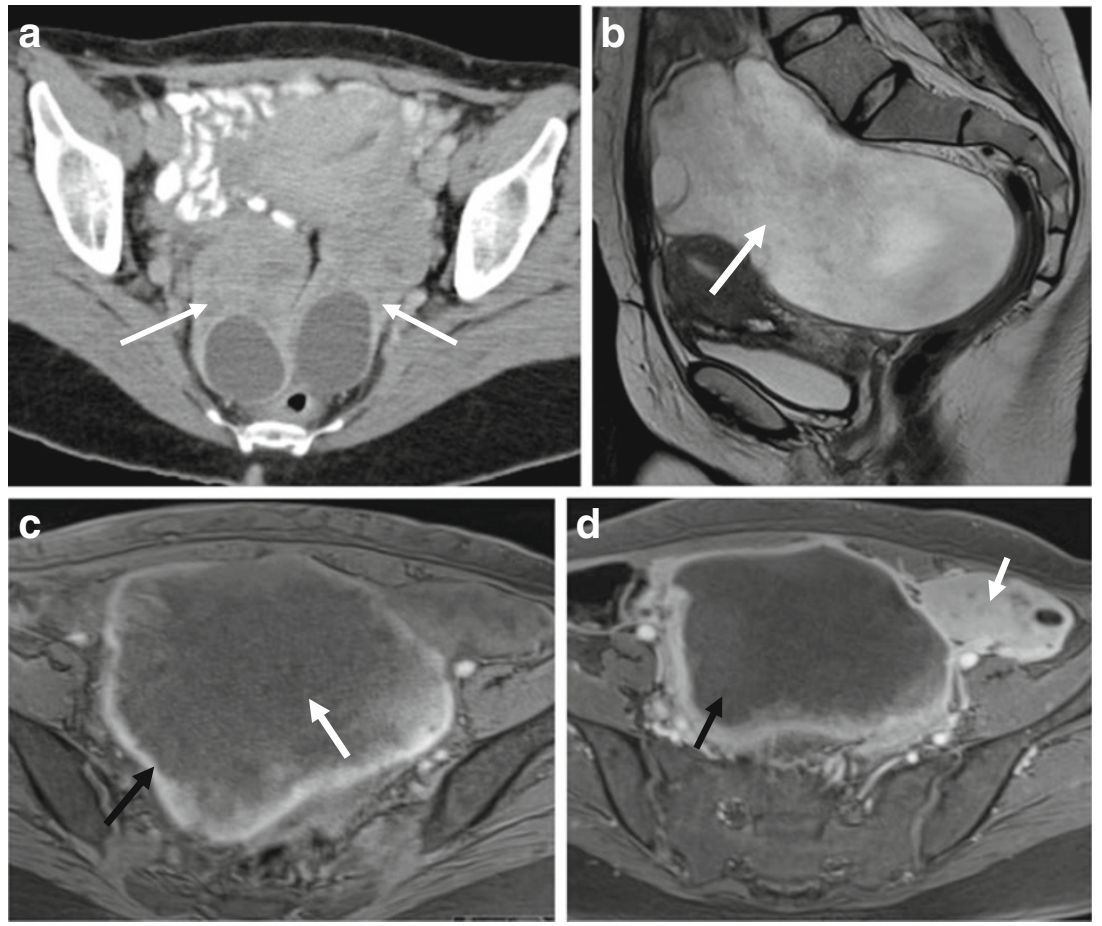

Fig. 4 Torsion of an ovarian mass. Patient with a history of gastric cancer developed lower abdominal discomfort and attended for CT (a), which demonstrates bilateral solid/cystic complex adnexal masses consistent with ovarian metastases. Two months later she presented to the emergency department with acute onset of right iliac fossa pain with nausea and MRI of the pelvis was performed (b-d). b Sagittal T2-weighted image demonstrates marked enlargement of the right ovary with high $\mathrm{T} 2$ signal intensity in keeping with stromal oedema (white arrow). c Axial T1 image with fat saturation shows central low signal intensity (white arrow) surrounded a rim of high signal intensity in the enlarged right ovary consistent with peripheral haemorrhage (black arrow). d Axial T1 fat sat image following gadolinium administration confirms lack of enhancement of the right ovary (black arrow consistent with right ovarian torsion). The left ovarian metastasis enhances avidly (white arrow) 
and give an appearance referred to as the "ground glass pattern" (Fig. 3a). In contrast to haemorrhagic cysts, endometriomas tend to be multiple and have a more stable appearance over time [20].

Presentation with a ruptured endometriotic cyst is uncommon but significant as emergency surgery may be required due to severe abdominal pain caused by peritoneal irritation resulting from the flow of the contents of the cyst [21]. Ruptured endometriotic cysts typically are multilocular or bilateral ovarian cysts with a thick wall and have loculated ascites confined to the pelvic cavity with pelvic fat infiltration on CT [22].

On MRI, T1 hyperintense cysts with T2 shading or multiple $\mathrm{T} 1$ hyperintense cysts regardless of the $\mathrm{T} 2$ signal intensity increase the sensitivity and specificity of diagnosis [23] (Figs. 3b, c).

\section{Adnexal torsion}

Adnexal torsion is the rotation of at least one turn of the ovaries, adnexa or the fallopian tube around the line of the tubo-ovarian ligament and the infundibulopelvic ligament [24]. It is a diagnosis that should be considered when evaluating female patients presenting to the emergency services with lower abdominal pain. Risk factors for the development of adnexal torsion include ovarian tumours (Fig. 4a) and ovarian cysts. It is important that adnexal torsion is diagnosed early as delay can lead to complications such as loss of adnexa or the ovary with the associated fertility problems. In rare cases, diagnostic delay can cause peritonitis or fatal thrombophlebitis. Initial misdiagnosis of adnexal torsion is common and studies have shown that only $23 \%$ to $66 \%$ of cases are given the correct presurgical diagnosis $[25,26]$.

Ultrasound, which is frequently used as the initial imaging investigation, may detect an adnexal mass, often in a relatively midline position. However, between $9 \%$ and $26 \%$ of adnexal torsions occurs in normal-sized ovaries and have normal appearances on ultrasound [27-29]. The use of Doppler sonography can be used to diagnose adnexal torsion. Doppler does this by detecting the absence of blood flow to the torted ovary. A characteristic "whirlpool sign" may be seen on colour Doppler where a corkscrew appearance of a twisted vascular pedicle is apparent. However, studies have found that although Doppler sonography has a high specificity for diagnosing adnexal torsion, it is not sensitive, missing the diagnosis up to $60 \%$ of the time [30, 31]. Arterial blood flow may be seen in adnexal torsion for several reasons, leading to false-negative US. Firstly, torsion may be intermittent. Secondly, only one of the two adnexal arteries (uterine and ovarian) may be torted. Thirdly, venous thrombosis secondary to torsion may lead to ovarian necrosis before arterial thrombosis occurs [28].
CT and MRI may demonstrate a twisted vascular pedicle and thickened fallopian tube in subacute torsion, and identify an underlying mass. Features of torsion using these modalities include eccentric or concentric wall thickening of the torted adnexal mass, fallopian tube thickening, uterine deviation to the effected side and ascites [32]. There may also be eccentric or diffuse poor contrast enhancement of the internal solid component or thickened wall [33] (Fig. 4d).

When using MRI to evaluate an indeterminate adnexal lesion following US, it is recommended that sequences include a $\mathrm{T} 1$ and fat-suppressed $\mathrm{T} 1$-weighted sequence to
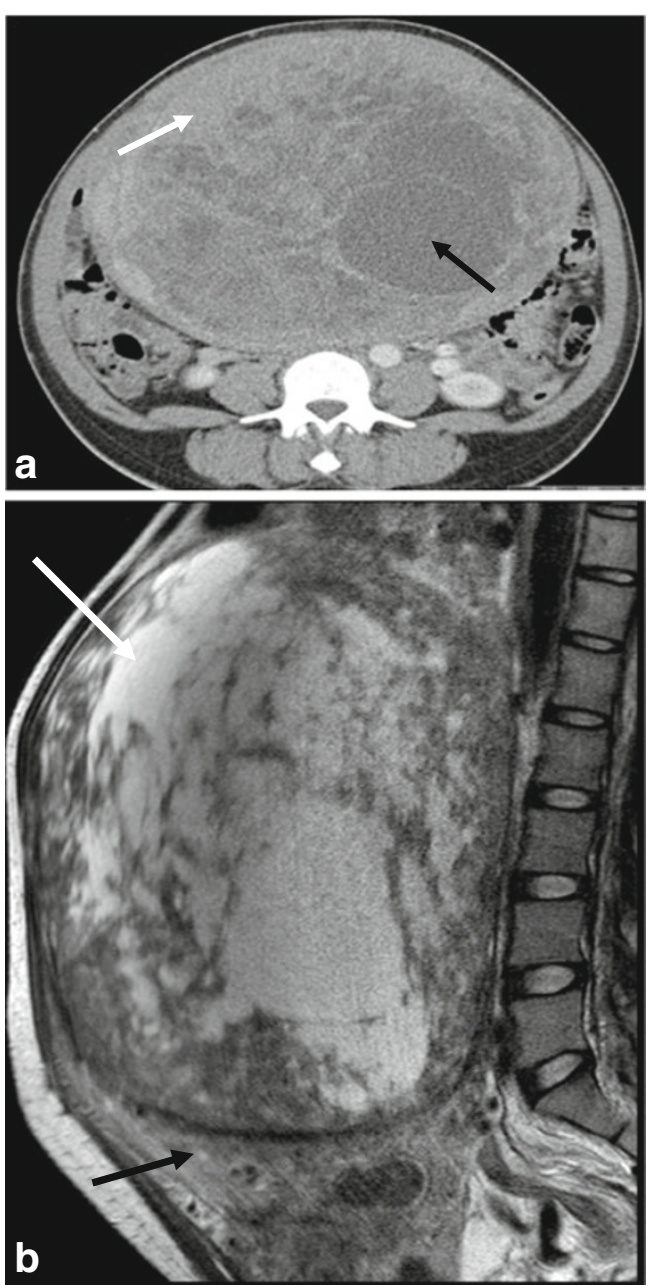

Fig. 5 Cystic fibroid degeneration. This patient presented to the emergency department with vaginal bleeding and lower abdominal pain. a CT following iv contrast administration shows demarcated regions of low density within the fibroid representing cystic degeneration (black arrow) with enhancing surrounding soft tissue (white arrow). These features are in keeping with degenerative change of a uterine fibroid. The differential diagnosis includes a complex ovarian mass. b Sagittal T2 MRI demonstrates a large heterogenous mass (white arrow) arising from the fundus of the uterus (black arrow). Cystic areas of degeneration are demonstrated by areas of high signal intensity within the fibroid. Identifying a connection to the uterus is important in making the correct diagnosis 
detect haemorrhage (Fig. 4c), which is a less common feature of adnexal torsion [32]. Contrast-enhanced, fatsuppressed, T1-weighted images can be used to detect absence of vascular supply (Fig. 4d). It should be remembered that haemoperitoneum and haemorrhage with ovarian masses can be demonstrated in other acute emergencies such as a haemorrhagic cysts and rupture. Sagittal MR imaging may be used to detect tubular protrusion from an adnexal mass towards the inferior uterus. This feature can be a cardinal sign of tube thickening. MRI can also detect smooth wall thickening of a twisted ovarian mass in cases of adnexal torsion [32]. Other MRI features that can suggest haemorrhagic infarction due to torsion include a thickened cyst wall and low enhancement in the solid component of the twisted ovarian mass $[14,32]$. The ovary may be grossly enlarged due to oedema (Fig. 4b).

\section{Fibroids: acute presentations}

Fibroids (leiomyoma) are the most common pelvic tumours affecting females in the fertile age group. They occur in 20 $40 \%$ of females above 30 years of age. These benign smooth muscle tumours are sensitive to oestrogens, which are thought to be a main contributory factor to fibroid growth over time. Symptoms usually manifest during the 4 th decade [14]. As fibroids may cause acute pain, patients may present at the emergency department. Acute pain may be caused by the degeneration of a fibroid when it outgrows its blood supply, torsion of a pedunculated fibroid or prolapse of a submucosal fibroid. Red (or haemorrhagic) degeneration is another type of acute fibroid degeneration seen in pregnancy and in patients on the oral contraceptive pill, caused by thrombosis of the venous outflow and resulting in a rapid increase in the size of the fibroid with acute haemorrhagic infarction. Acute complications of fibroids are rarely seen but may be serious. Acute bleeding into fibroids can lead to hypovolaemic shock and cause the death of the patient [34, 35].

Ultrasound is often the initial diagnostic imaging modality for suspected complications of fibroids. A simple fibroid is usually seen as a hypoechoic lesion that may be well defined and arising within the surrounding myometrium. Fibroids can also demonstrate posterior acoustic enhancement or attenuation without any calcification. Degeneration of fibroids gives a more complex US appearance with areas of cystic change and Doppler can show circumferential vascularity. Fibroids that are torted or are necrotic will show absence of flow on Doppler US.

In acute presentation of pain, fibroid degeneration on CT may show a cystic appearance of a fibroid mass, with reduced enhancement and hypodense areas. Fibroid degeneration can often be difficult to distinguish from cystic ovarian masses on CT and MRI is often needed to distinguish between them (Fig. 5a, b). MRI can be very helpful in this respect, when investigating suspected acute fibroid complications (Fig. 6a-c). The use of multiplanar views can enable localisation of fibroids and can make it possible to distinguish fibroids from ovarian masses. Fibroids that have undergone degeneration show a great diversity in their MRI appearances with cystic change and areas of nonenhancement. In cases of red degeneration, the patient often presents with an acute abdomen. MRI appearances show high signal intensity centrally within the fibroid on T1-weighted images consistent with blood, with reduced signal at the periphery on T2-weighted images secondary to haemosiderin deposition (Fig. 6a, b). There may be heterogeneous signal intensity on $\mathrm{T} 2$, with no enhancement post-gadolinium administration (although gadolinium is not given to pregnant patients) $[36,37]$.

Submucosal pedunculated leiomyomas may prolapse through the cervical canal and even the vaginal canal, and
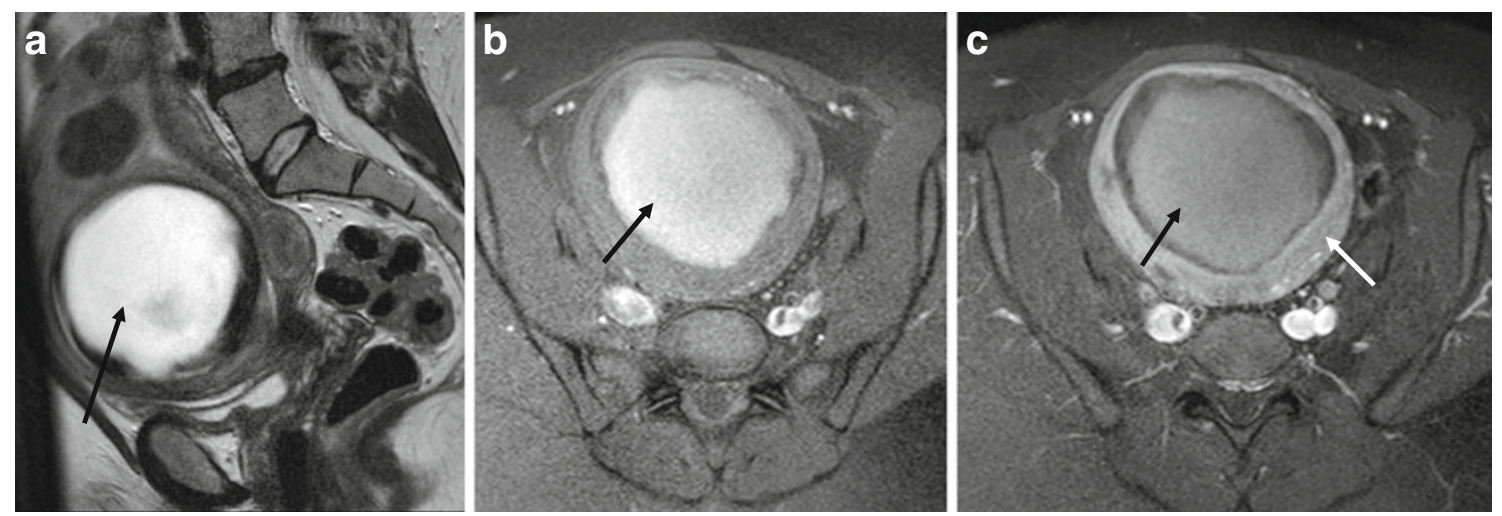

Fig. 6 Haemorrhagic fibroid degeneration. This patient, known to have uterine fibroids, presented to the accident and emergency department with low-grade pyrexia, tachycardia and acute lower abdominal pain. a Sagittal T2 image demonstrates a large uterine fibroid with high signal intensity centrally with a very low signal intensity rim suggestive of peripheral haemosiderin. b Axial T1 with fat-saturated image shows high signal intensity within the fibroid consistent with haemorrhage (black arrow). c Axial T1 with fat saturation following gadolinium administration demonstrates lack of enhancement within the fibroid (black arrow), consistent with infarction. The surrounding myometrium enhances normally (white arrow) 

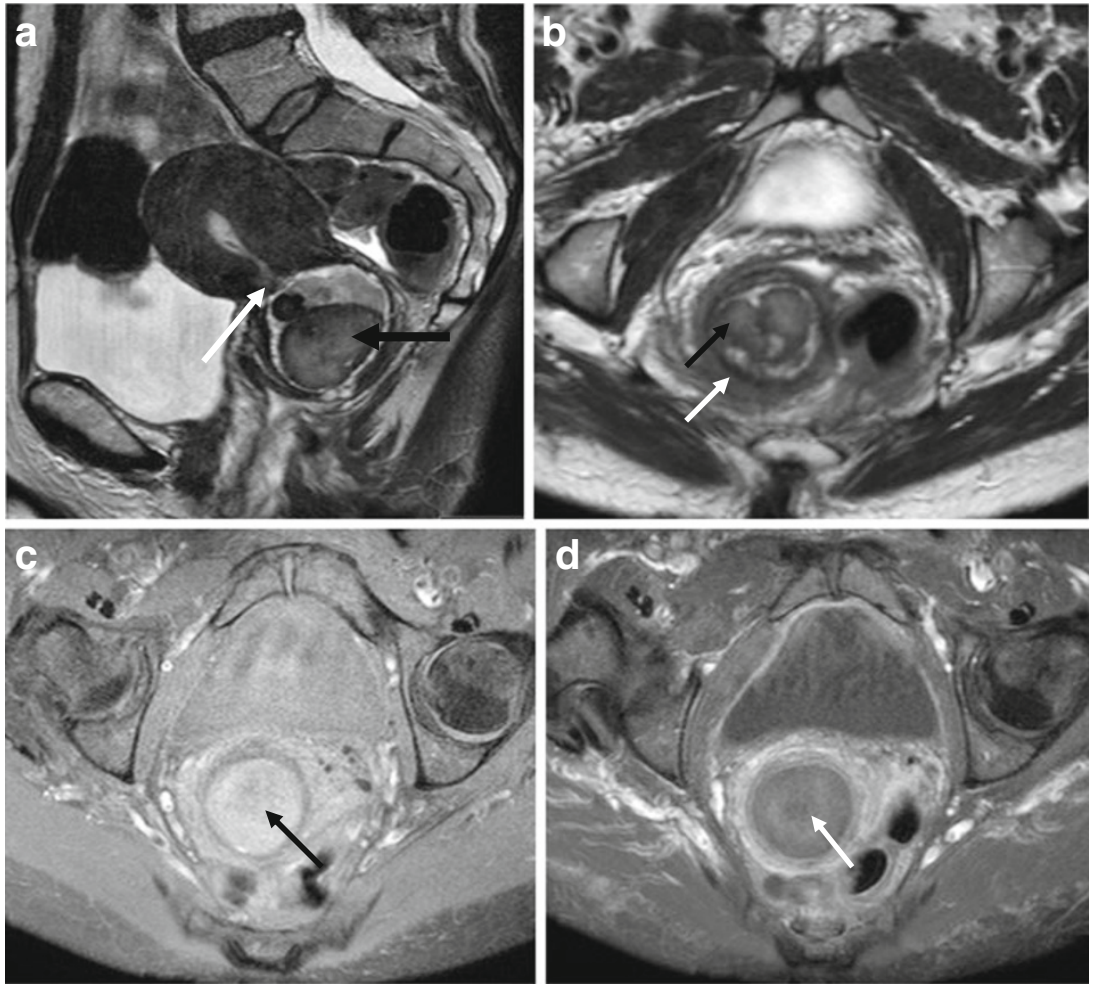

Fig. 7 Pedunculated submucosal fibroid with prolapse and torsion. The patient presented to the emergency department with acute abdominal pain and vaginal bleeding. a Sagittal T2 image demonstrates a fibroid arising on a stalk (white arrow) that originates in the lower endometrial cavity. The fibroid has prolapsed into the endocervical canal (black arrow) and demonstrates areas of low T2 suggestive of haemorrhage. These features are typical of a pedunculated fibroid or

typically present with pain and abnormal vaginal bleeding. A pedunculated submucosal leiomyoma may be detected on clinical gynaecological examination if there is prolapse into the vaginal canal. The role of imaging is in diagnosis of the polyp. b Axial T2 image demonstrates the torted fibroid (white arrow) surrounded by the ring of cervical stroma (black arrow). c Axial T1 fatsaturated image demonstrates high signal intensity within the fibroid indicating haemorrhage (black arrow). d Axial T1 fat-saturated image following gadolinium administration demonstrates lack of enhancement consistent with torsion (white arrow)

lesion origin and identification of the stalk and the uterine attachment of the leiomyoma. MRI is the most effective imaging modality in the diagnosis of a prolapsed leiomyoma [38]. Sagittal T2-weighted images demonstrate the prolapsing

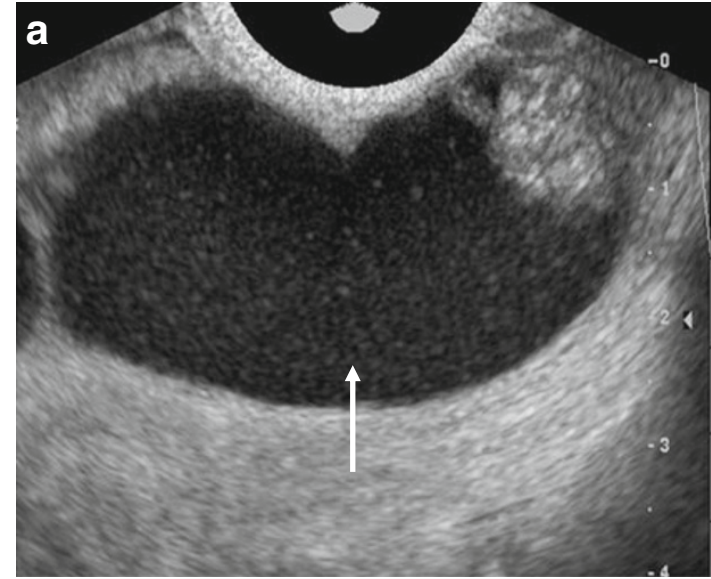

Fig. 8 Pelvic inflammatory disease with pyosalpinx on ultrasound. This patient presented to the emergency department with lower abdominal pain, pyrexia and vomiting. a-b Transvaginal ultrasound of both adenexa. There are bilateral adenexal cysts that contain low-level

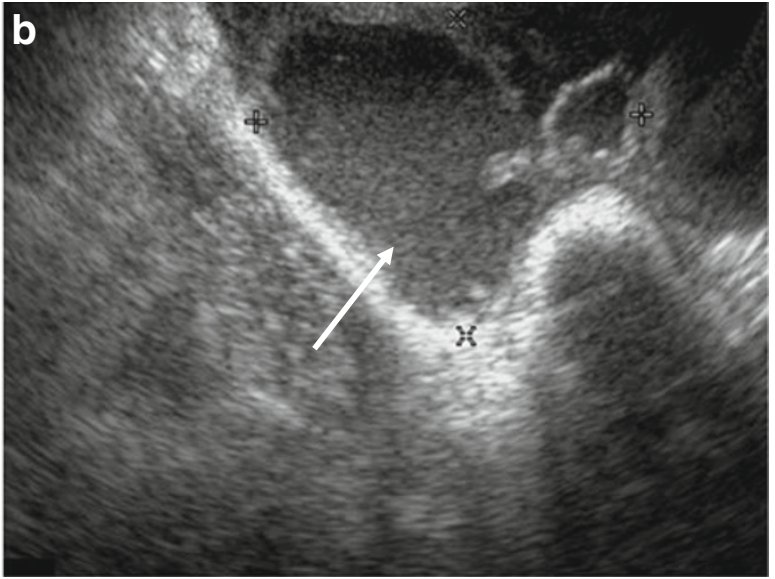

echogenic material and have a tubular configuration (white arrows). The appearance is in keeping with bilateral pyosalpinges, a complication of pelvic inflammatory disease 
leiomyoma extending into the endocervical canal, and frequently show the stalk and its uterine attachment (Fig. 7a). The stalk extends up into the endometrial cavity and typically has multiple linear structures running through it, and the appearance of the stalk and prolapsed fibroid has been described as the 'broccoli sign' [36]. The fibroid may become torted and haemorrhagic, and shows heterogeneous signal intensity on MRI, with lack of enhancement following contrast administration (Fig. 7). MRI can provide important clinical information for pre-surgical planning [38, 39].

CT may show a heterogeneously enhancing mass prolapsing into the vagina and perineum. Often the mass can show diffuse low attenuation, which indicates necrosis of the fibroid.

\section{Pelvic inflammatory disease}

Pelvic inflammatory disease (PID) is described as a spread of inflammation from the endometrial cavity and fallopian tubes into the pelvis. It is an umbrella term, which encompasses endometritis, salpingitis and tubo-ovarian abscesses. It usually affects women in the reproductive age group and accounts for $25 \%$ of visits to the emergency departments with gynaecological pain. The symptoms of pelvic inflammatory disease are general aching pain in the pelvis that varies in severity. It is also common for sufferers to have vaginal discharge and cervical excitation on examination. Patients often have a leukocytosis, increased inflammatory markers and may be febrile. A long course of antibiotic treatment is the most common treatment of PID. Complications of untreated PID can have serious implications for the patient and include infertility, ectopic pregnancy, chronic abdominal pain and the development of tubo-ovarian abscesses requiring surgical intervention. Organisms such as sexually transmitted Chlamydia trachomatas and Nissera gonorrhoea are causative agents in developing ascending cervicitis. Other conditions that may lead to the development of tubo-ovarian abscess are diverticulitis, appendicitis and tuberculosis [37, 40].

In the early phase of infection, it is common for ultrasound and $\mathrm{CT}$ appearances to be normal. As the disease advances, transabdominal ultrasound can demonstrate uterine enlargement and thickening of the endometrium. Ultrasound can also show the loss of tissue plains and an ill-defined uterus. Hydrosalpinx or pyosalpinx is a common complication of salpingitis. Ultrasound can identify dilated fallopian tubes containing heterogenous fluid with echogenic debris; features typical of pyosalpinx. The fallopian tubes may be folded and demonstrate areas of tube tapering, and intraluminal small linear echogenic foci may be visualised (Fig. 8a, b). As pyosalpinx develops into tubo-ovarian abscesses, echogenic debris can be seen in the fallopian tubes and ovaries, representing inflammatory exudates, blood and pus [37].
In order to reduce exposure to radiation, $\mathrm{CT}$ is not usually employed as a first-line imaging investigation in cases of suspected PID. The CT appearances of PID are often non-specific with parapelvic fat stranding, a small volume of free fluid and thickened uterosacral ligaments. However, the development of tubo-ovarian abscesses results in thickened irregularly enhancing complex adnexal masses with thick walls and septations containing complex fluid collections (Fig. 9). Tubular configurations are usually indicative of pyosalpinx. Difficulty in distinguishing tubo-ovarian from other pelvic abscesses can be difficult. Tubo-ovarian abscesses frequently cause anterior displacement of the broad ligament as the mesovarium

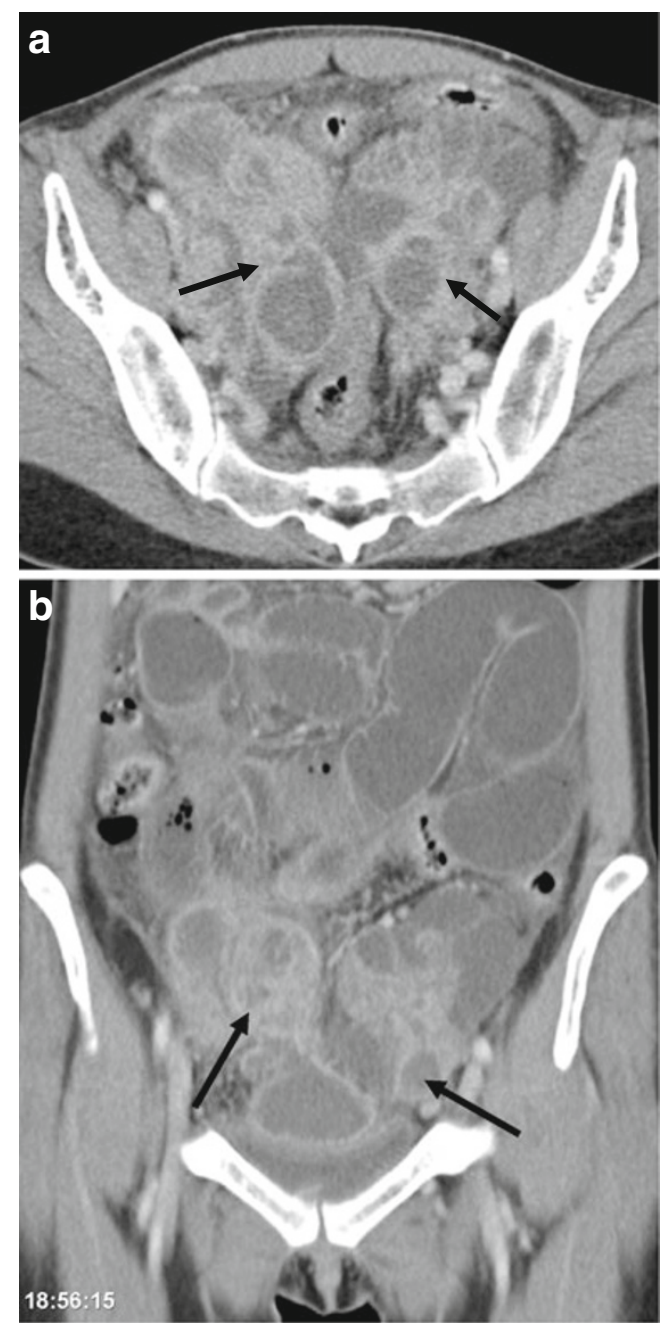

Fig. 9 Pelvic inflammatory disease with pyosalpinx on CT. This patient presented to the accident and emergency department with abdominal pain and pyrexia. She had a raised white cell count and CRP. The clinicians suspected an intra-abdominal collection. a CT demonstrates bilateral adnexal complex fluid-filled and thick-walled cysts typical for tubo-ovarian abcess formation, a complication of pelvic inflammatory disease. b Coronal reformat of the CT demonstrates bilateral tubo-ovarian abcesses as well as distention of multiple bowel loops due to an associated ileus 
is positioned more posteriorly, and this can assist in making the diagnosis [37].

On MRI, inflammation in the parametrium may be seen as ill-defined hyperintense areas on T2-weighted fat-suppressed images, in addition to enhancement on gadolinium-enhanced T1-weighted images [37]. A pyosalpinx can be visualised as a dilated, fluid-filled, tortuous $\mathrm{C}$ or S-shaped structure. Thickwalled fluid-filled abscesses and pyosalpinx may have heterogeneous signal intensity on both $\mathrm{T} 1$ and $\mathrm{T} 2$ weighting due to mixtures of pus, haemorrhage and debris. The thick-walled mass typically demonstrates marked enhancement following iv gadolinium administration (Fig. 10c). Ovarian cancer should always be considered when investigating a possible tubo-ovarian mass. Although radiological features of abscess and ovarian malignancy can be similar, the clinical presentation plays an important role in establishing the diagnosis and follow-up imaging following treatment confirms decreasing size of adnexal massses [40, 41].

\section{Conclusion}

In cases of suspected acute gynaecological disease, the findings on imaging must be interpreted in association with the clinical presentation. The pregnancy status of the patient must always be established, in order to exclude ectopic pregnancy and to avoid using imaging modalities, which pose a risk to the foetus. Clinical signs of sepsis can make the clinicians more suspicious of PID. Haemorrhagic ovarian cysts give a characteristic internal reticular pattern on ultrasound with high attenuation contents on non-contrast CT, an enhancing cystic wall and contrast-enhanced blood in the pelvis on delayed phases in cases of cyst rupture (Table 1). Although endometriotic cysts rarely cause an acute presentation, it is important to recognise the groundglass appearance on ultrasound and characteristic high signal intensity on T1- and shading on T2-weighted images on MRI. Where adnexal torsion is suspected, Doppler can demonstrate characteristic whirlpool or corkscrew signs of a twisted vascular pedicle. Contrast-enhanced CT and MRI may demonstrate reduced enhancement in the twisted ovarian mass, another diagnostic feature of torsion (Table 1). Where torsion is suspected clinically but diagnostic imaging features are not demonstrated, it should be remembered that imaging cannot be relied upon to confirm the diagnosis. MRI is the most effective modality for characterisation of fibroid degeneration. Red degeneration demonstrates high signal intensity centrally on T1-weighted images due to acute blood and a low signal periphery on T2 due to haemosiderin deposition. MRI appearances of submucosal pedunculated leiomyomas can demonstrate a "broccoli sign" with heterogeneous signal due to the characteristic stalk linking the herniating fibroid with the endometrial cavity (Table 1). This type of fibroid is at particular risk of torsion. Complications of PID can be demonstrated with ultrasound, with dilated fallopian tubes with internal echogenic debris being a typical feature of pyosalpinx. Although CT and MRI are not routinely used to investigate PID complications, these modalities may demonstrate typically bilateral dilated fallopian tubes and inflammatory changes in the adjacent fat planes (Table 1). In most presentations of gynaecological emergencies, US, and occasionally CT, can be the simplest and quickest way to assess the patient, with MRI reserved for further characterisation of complex masses and problemsolving. Overlap in the presenting features of acute gynaecologic disease and gastrointestinal disease remains a significant challenge in the emergency department and imaging plays an important diagnostic role.
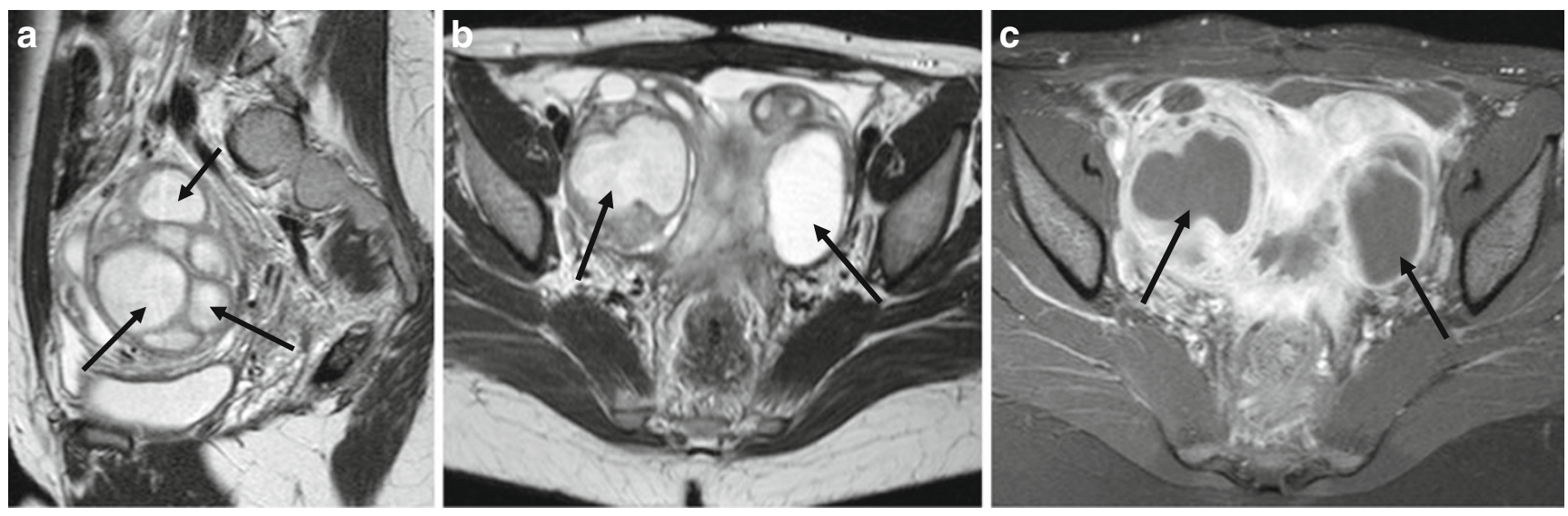

Fig. 10 Pelvic inflammatory disease with pyosalpinx on MRI. This patient presented to the emergency department with pyrexia, lower abdominal pain and diarrhoea. a Sagittal T2 image of the pelvis demonstrates multiple fluid-filled cystic structures within the right adnexa (black arrows). The complex cyst is thick walled and there is adjacent fat stranding. b Axial
T2 image demonstrates bilateral tubo-ovarian abcesses. c Axial T1 fatsaturated image following gadolinium administration demonstrates low signal intensity within the pus-filled cavities and marked enhancement of the inflammatory walls. The imaging appearances may overlap with ovarian malignancy but the clinical presentation is of sepsis 


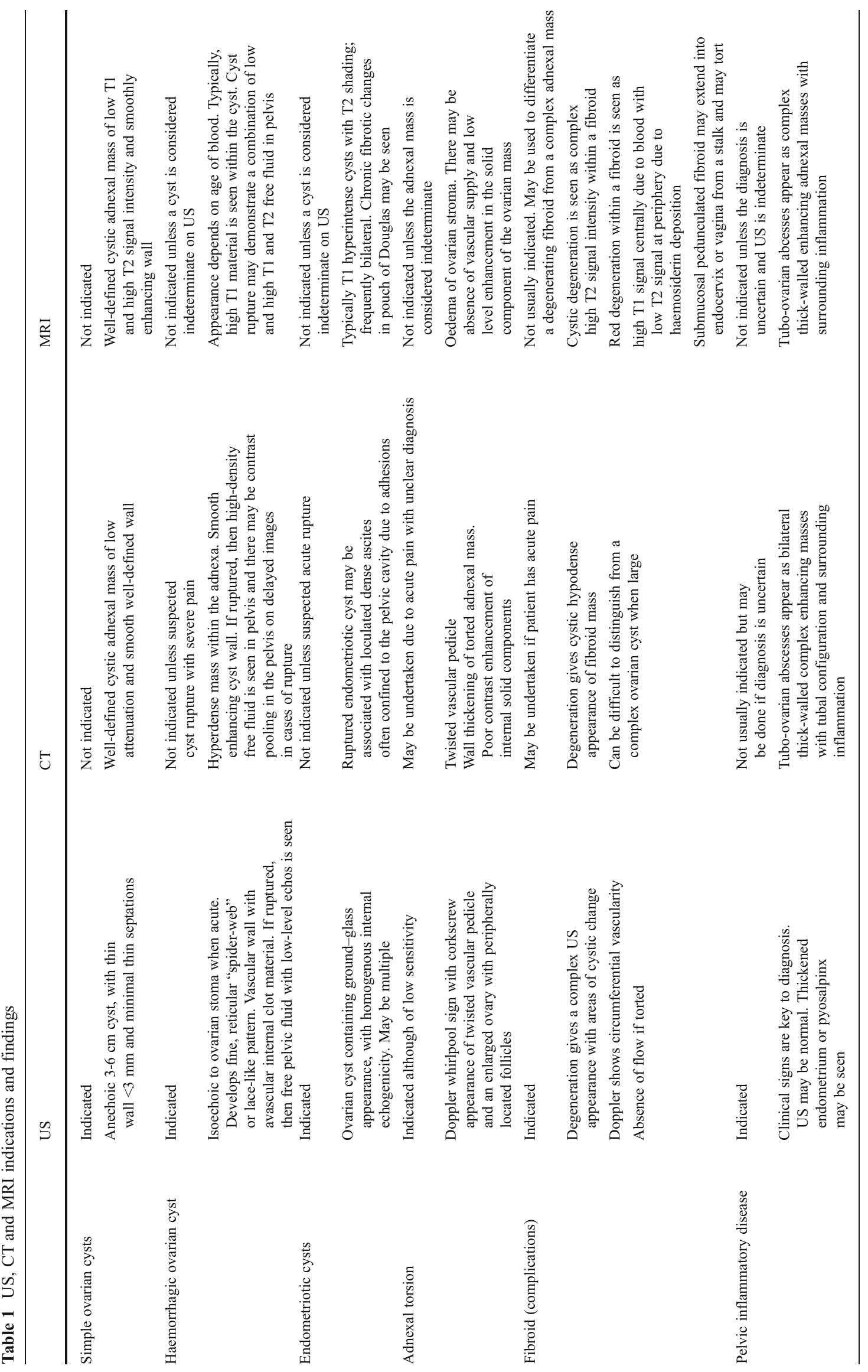




\section{References}

1. Stenchever M (2001) Comprehensive gynaecology. 2nd edn. Mosby

2. Vandermeer FQ, Wong-You-Cheong JJ (2009) Imaging of acute pelvic pain. Clin Obstet Gynecol 52:2-20

3. Ogawa Y, Fukuda T, Matsunaga N et al (1991) Computed tomographic features in ovarian bleeding. Nihon Igaku Hoshasen Gakkai Zasshi 51:394-399

4. Hertzberg BS, Kliewer MA, Bowie JD et al (1999) Adnexal ring sign and hemoperitoneum caused by hemorrhagic ovarian cyst: pitfall in the sonographic diagnosis of ectopic pregnancy. AJR Am J Roentgenol 173:1301-1302

5. Kaakaji Y, Nghiem HV, Nodell C, Winter TC (2000) Sonography of obstetric and gynecologic emergencies: Part II, Gynecologic emergencies. AJR Am J Roentgenol 174:651-656

6. Kaakaji Y, Nghiem HV, Nodell C, Winter TC (2000) Sonography of obstetric and gynecologic emergencies: Part I, Obstetric emergencies. AJR Am J Roentgenol 174:641-649

7. Jeong YY, Outwater EK, Kang HK (2000) Imaging evaluation of ovarian masses. Radiographics 20:1445-1470

8. Valentin L (2004) Use of morphology to characterize and manage common adnexal masses. Best Pract Res Clin Obstet Gynaecol 18:71-89

9. Borders RJ, Breiman RS, Yeh BM, Qayyum A, Coakley FV (2004) Computed tomography of corpus luteal cysts. J Comput Assist Tomogr 28:340-342

10. Bennett GL, Slywotzky CM, Giovanniello G (2002) Gynecologic causes of acute pelvic pain: spectrum of CT findings. Radiographics 22:785-801

11. Nishino M, Hayakawa K, Iwasaku K, Takasu K (2003) Magnetic resonance imaging findings in gynecologic emergencies. J Comput Assist Tomogr 27:564-570

12. Kataoka ML, Togashi K, Inoue T, Fujii S, Konishi J (1999) Evaluation of ectopic pregnancy by magnetic resonance imaging. Hum Reprod 14:2644-2650

13. Busard MP, Pieters-van den Bos IC, Mijatovic V, Van Kuijk C, Bleeker MC, van Waesberghe JH (2011) Evaluation of MR diffusion-weighted imaging in differentiating endometriosis infiltrating the bowel from colorectal carcinoma. Eur J Radiol Sept 9 [Epub ahead of print]

14. Webb EM, Green GE, Scoutt LM (2004) Adnexal mass with pelvic pain. Radiol Clin North Am 42(2):329-348

15. Ding DC, Chen SS (2005) Conservative laparoscopic management of ovarian teratoma torsion in a young woman. J Chin Med Assoc 68:37-39

16. Kalish GM, Patel MD, Gunn ML, Dubinsky TJ (2007) Computed tomographic and magnetic resonance features of gynecologic abnormalities in women presenting with acute or chronic abdominal pain. Ultrasound Q 23:167-175

17. Comerci JT, Licciardi F, Bergh PA, Gregori C, Breen JC (1994) Mature cystic teratoma: a clinicopathologic evaluation of 517 cases and review of the literature. Obstet Gynecol 84:22-28

18. Outwater EK, Siegelman ES, Hunt JL (2001) Ovarian teratomas: tumor types and imaging characteristics. Radiographics 21:475-490

19. Bis KG, Vrachliotis TG, Shetty AN, Maximovich A, Hricak H (1997) Pelvic endometriosis: MR imaging spectrum with laparoscopic correlation and diagnostic pitfalls. Radiographics 17:639-655
20. Porpora MG, Gomel V (1997) The role of laparoscopy in the management of pelvic pain in women of reproductive age. Fertil Steril 68:765-779

21. Coulier B, Malbecq S, Brinon PE, Ramboux A (2008) MDCT diagnosis of ruptured tubal pregnancy with massive hemoperitoneum. Emerg Radiol 15:179-182

22. Lee YR (2011) CT imaging findings of ruptured ovarian endometriotic cysts: emphasis on the differential diagnosis with ruptured ovarian functional cysts. Korean J Radiol 12:59-65

23. Togashi K, Nishimura K, Kimura I (1991) Endometrial cysts: diagnosis with MR imaging. Radiology 180:73-78

24. Huchon C, Fauconnier A (2010) Adnexal torsion: a literature review. Eur J Obstet Gynecol Reprod Biol 150:8-12

25. Haskins T, Shull BL (1986) Adnexal torsion: a mind-twisting diagnosis. South Med J 79:576-577

26. Hibbard LT (1985) Adnexal torsion. Am J Obstet Gynecol 152: 456-461

27. Bider D, Mashiach S, Dulitzky M, Kokia E, Lipitz S, Ben-Rafael Z (1991) Clinical, surgical and pathologic findings of adnexal torsion in pregnant and nonpregnant women. Surg Gynecol Obstet 173:363-366

28. Chang HC, Bhatt S, Dogra VS (2008) Pearls and pitfalls in diagnosis of ovarian torsion. Radiographics 28:1355-1368

29. Mage G, Canis M, Menhes H, Pouly JL, Bruhat MA (1989) Laparoscopic management of adnexal torsion. A review of 35 cases. J Reprod Med 34:520-524

30. Pena JE, Ufberg D, Cooney N, Denis AL (2000) Usefulness of Doppler sonography in the diagnosis of ovarian torsion. Fertil Steril 73:1047-1050

31. Lee EJ, Kwon HC, Joo HJ, Suh JH, Fleischer AC (1998) Diagnosis of ovarian torsion with color Doppler sonography: depiction of twisted vascular pedicle. J Ultrasound Med 17:83-89

32. Rha SE, Byun JY et al (2002) CT and MR imaging features of adnexal torsion. Radiographics 22:283-294

33. Lee JH, Park SB, Shin SH (2009) Value of intra-adnexal and extraadnexal computed tomographic imaging features diagnosing torsion of adnexal tumor. J Comput Assist Tomogr 33:872-876

34. Vercellini P, Maddalena S, de Giorgi O, Aimi G, Crosignani PG (1998) Abdominal myomectomy for infertility: a comprehensive review. Hum Reprod 13:873-879

35. Neuwirth RS (1995) Hysteroscopic submucous myomectomy. Obstet Gynecol Clin North Am 22:541-558

36. Ueda H, Togashi K, Konishi I (1999) Unusual appearances of uterine leiomyomas: MR imaging findings and their histopathologic backgrounds. Radiographics 19 Spec No: S131-145

37. Dohke M, Watanabe Y, Okumura A (2000) Comprehensive MR imaging of acute gynecologic diseases. Radiographics 20:15511566

38. Wilde S, Scott-Barrett S (2009) Radiological appearances of uterine fibroids. Indian J Radiol Imaging 19:222-231

39. Kim JW, Lee CH, Kim KA, Park CM (2008) Spontaneous prolapse of pedunculated uterine submucosal leiomyoma: usefulness of broccoli sign on CT and MR imaging. Clin Imaging 32:233-235

40. Tukeva TA, Aronen HJ, Karjalainen PT, Molander P, Paavonen T, Paavonen J (1999) MR imaging in pelvic inflammatory disease: comparison with laparoscopy and US. Radiology 210:209-216

41. Sam JW, Jacobs JE, Birnbaum BA (2002) Spectrum of CT findings in acute pyogenic pelvic inflammatory disease. Radiographics 22:1327-1334 